\title{
Signposts for CLARIN
}

\author{
Denis Arnold \\ Leibniz-Institut für Deutsche Sprache (IDS) \\ Mannheim, Germany \\ arnoldeids-mannheim.de
}

\section{Bernhard Fisseni \\ IDS and}

Universität Duisburg-Essen, Germany

bernhard.fisseni@uni-due.de

\author{
Thorsten Trippel \\ Eberhard Karls Universität \\ Tübingen, Germany \\ thorsten.trippel@uni-tuebingen.de
}

\begin{abstract}
An implementation of CMDI-based signposts and its use is presented in this paper. Arnold, Fisseni et al. (2020) present signposts as a solution to challenges in long-term preservation of corpora. Though applicable to digital resources in general, we focus on corpora, especially those that are continuously extended or subject to modification, e.g., due to legal injunctions, but also may overlap with respect to constituents, and may be subject to migrations to new data formats. We describe the contribution signposts can make to the CLARIN infrastructure, notably virtual collections, and document the design for the CMDI profile.
\end{abstract}

\section{Introduction}

The current paper presents an implementation of the concept of signposts (Arnold, Fisseni et al. 2020) which is based on the Component Metadata Infrastructure (CMDI, see Broeder et al. 2012), and explains how signposts can contribute to the overall CLARIN infrastructure. The contribution concerns the use of persistent identifiers (PIDs) for resources, and the handling of data removal, data migrations and versioning as well as deduplication.

A signpost is a metadata file for a leaf on the tree of resources, for instance a single text or an audio recording. Using terminology from the area of long-term archival, we distinguish conceptual object (CO) from logical object (LO) (see chapter 9 by Stefan Funk in Neuroth et al. 2009). ${ }^{1}$ A CO can be realized in different LOs, for instance an audio recording (CO) can be realized in files of different audio formats (LO). A signpost represents a conceptual object (CO), and also refers to logical objects (LOs, typically at least one) belonging to it.

The most important point about signposts is that they change the idea what a PID refers to when providing data: While traditionally, PIDs may point directly to data files (LOs), ${ }^{2}$ it is suggested here that PIDs only refer to signposts (COs), and to leave it to signposts to point to files. The reason for this change is that LOs may be volatile, even if the represented information stays the same. By adding signposts as a layer of indirection, we can achieve an acceptable trade-off between the necessity of modifying data and the demands of long-term archival on the one hand and Open Science as well as reproducibility on the other.

Signposts are first motivated below with examples regarding growing corpora, i.e. large corpora that are constantly extended and contain material where the conglomerate of commercial interests, intellectual property rights and privacy rights constitutes a non-trivial problem. However, all aspects signposts address are relevant to other kinds of corpora as well, generally to a different degree. Moreover, not only customary, but also virtual collections in particular benefit from signposts, as will be discussed in section 4 . In case of small and 'legally' permanent corpora, signpost information may be included in the corpus metadata. Signposts replace the concept of tombstones, which are less flexible than signposts (see Arnold, Fisseni et al. 2020).

\section{Motivating Signposts}

The motivation for signposts comes from the impermanence of logical objects, specifically three aspects: the necessity of deletions due to legal actions, (conceptual) deduplication and data migration.

\footnotetext{
This work is licenced under a Creative Commons Attribution 4.0 International Licence. Licence details: http://creativecommons . org/licenses/by/4.0/

${ }^{1}$ Funk (chapter 9 in Neuroth et al. 2009) also distinguish the level of physical object which, however, is not immediately relevant for our current discussion.

${ }^{2}$ PIDs are also used to refer to datasets (see, e.g., De Smedt, Koureas and Wittenburg 2020 for a suggestion on how to structure datasets). However, we focus on data here.
} 


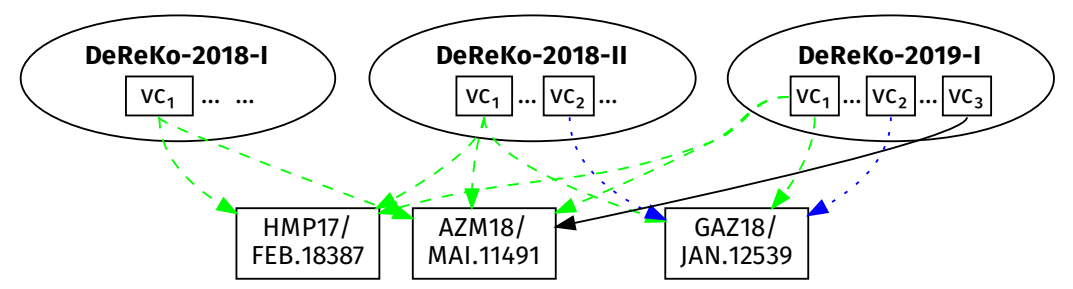

Figure 1: Relationship between DeReKo releases, virtual sub-corpora and texts (from Arnold, Fisseni et al. 2020). Texts may be part not of one, but many (virtual) corpora, and may belong to different versions of corpora.

Long-term Preservation vs. Legal Necessity of Deletions. In the realm of long-term preservation, we assume that original data, in the sense of COs, will always be retained. However, e.g. when building corpora from newspapers, it may become necessary to remove data from COs due to injunctions or revocation of licenses.

Migration. File formats may fall out of use, so that data must be converted to new formats, which in the OAIS model (CCSDS 2012) is called migration. Anecdotally consider the German Reference Corpus (DeReKo, see, e.g. Kupietz et al. 2010) compiled at the Leibniz Institute for the German Language (IDS). Between 1999 and 2005, SGML (ISO8879:1986 1986) / CES were used as its data format, then DeReKo was converted to XML (for the history and the decisions involved, see Lüngen and Sperberg-McQueen 2012), based on the TEI's P3 and later P5 recommendations (Sperberg-McQueen and Burnard 1999; Burnard and Bauman 2020). Similar conversions occurred in the IDS' oral corpora. Even if we assume that we retain the original LOs, which goes beyond the OAIS model, we would want to add new ones as time progresses. For instance, we want to provide XML files conforming to P5 today rather than P3. It may then be a good idea to retire the intermediate versions to avoid storage cost. With the traditional approach to metadata, these changes mean that we have to change the metadata in each of these steps. With signposts, we only change the signpost.

Complex Corpus Structures. Especially growing corpora may have intricate structures, e.g. overlapping with respect to COs. If information were recorded in the metadata of the parent structures of the leaf COs, the metadata records would have to be changed for several corpora, while with signpost only the latter must be adapted. Figure 1 shows the relationships between the DeReKo corpus releases and virtual corpora $v c_{1, \ldots, 3}$, and three texts. Based on release DeReKo-2018-I, $v c_{1}$ was defined, ${ }^{3}$ already containing the texts HMP17/FEB. 18387 and AZM18/MAI.11491. DeReKo-2018-II added GAZ18/JAN.12539 to $v c_{1}$. Based on DeReKo-2018-II, $v c_{2}$ was defined, containing the text GAZ18/JAN.12539. $v c_{3}$ was defined initially on DeReKo-2019-I, also containing AZM18/MAI.1149. This shows that texts in DeReKo may belong to many different corpora. In this case, removal becomes a complex matter.

In the next releases of both corpora in the IDS repository, we plan to implement signposts to avoid manually editing thousands of files in cases of conversion and legal issues. We will report on the first corpus successfully using signposts in section 5 .

\section{Signposts for CLARIN: A CMDI Profile for Signposts}

Reusing existing CMDI components, we developed a metadata profile for signposts, the signpost profile has the identifier clarin.eu:cr1:p_1587363818266 ${ }^{4}$ in the component registry.

The CMDI profile reuses existing components and intends to include technical information that can be automatically extracted based on a file. This information can be gained by the File Information Tool Set (FITS $)^{5}$ or other readily available tools to facilitate processing. The collected information includes the original filename, media type, file size in bytes, various checksums and cryptographic hashes. Besides this basic technical information on a LO, the signpost should also include information on the status of each LO, i.e.

\footnotetext{
${ }^{3}$ For the importance of virtual corpora in DeReKo's primordial sample design and extensionally or intensionally defined virtual corpora see Kupietz et al. (2010).

${ }^{4}$ see https://catalog.clarin.eu/ds/ComponentRegistry/rest/registry/1.1/profiles/clarin.eu:cr1:p_ $1587363818266 / 1.2 / x s d$, which is still in the development state, but accessible

${ }^{5}$ See http://fitstool.org
} 


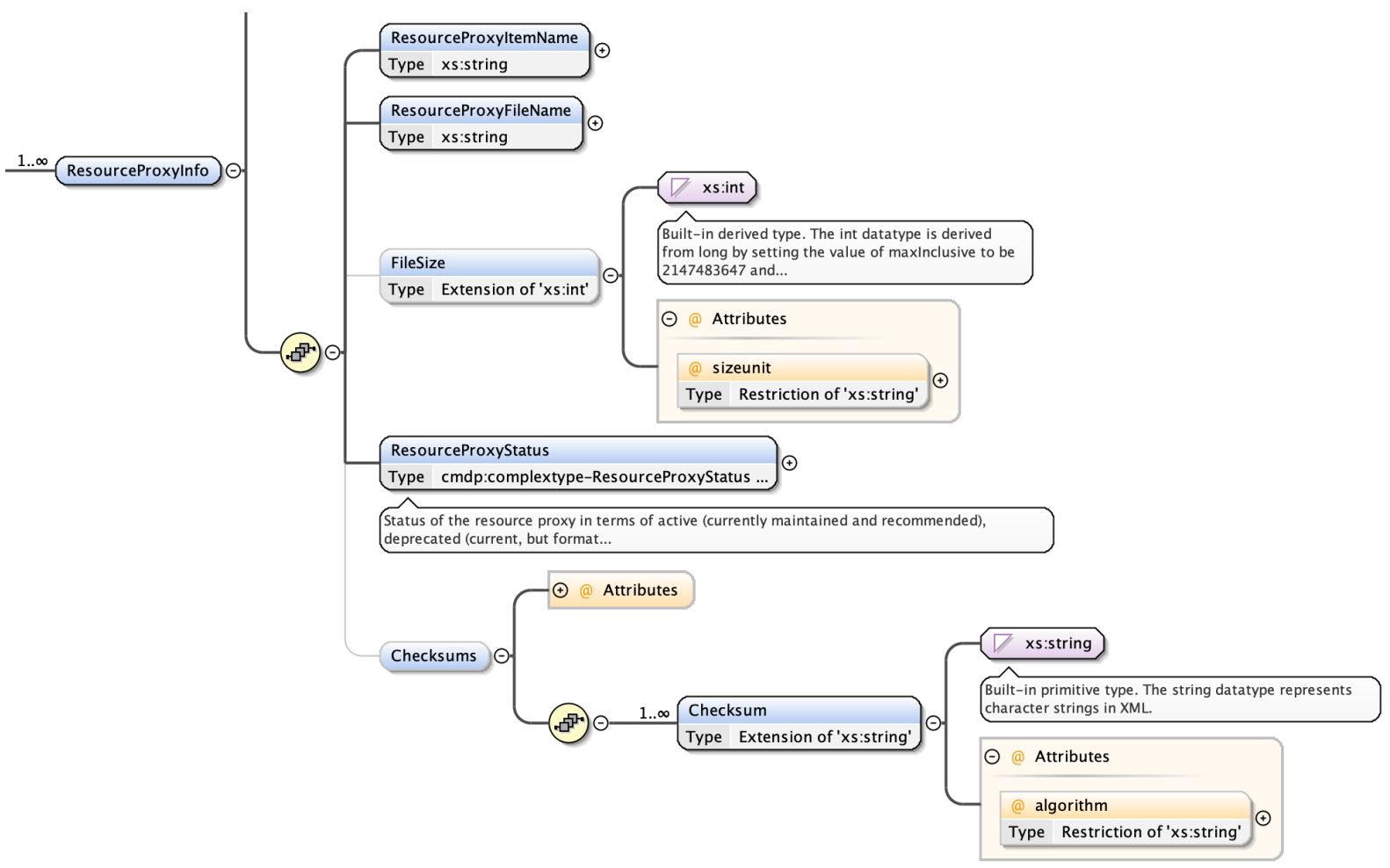

Figure 2: Visualisation of the structure for the metadata provided for each resource proxy in the CMDI Profile signpost (clarin.eu:cr1:p_1587363818266)

whether this is the currently maintained version, whether the use of the LO is deprecated (for example in the case of a migration to other data formats) or whether a file is no longer available. Reasons can be given in the provenance information (see next subsection). The schema also allows referring to a $\mathrm{LO}$ by a specific name that is not its filename, which is occasionally necessary.

The nature of the signpost profile is different from other profiles, in the sense that it is not intended to provide a meaningful description of a resource and does not foster findability by search engines such as the VLO. Hence it does - by design - neither cater for the VLO facets, nor respect quality criteria which are automatically evaluated by the Curation Module ${ }^{6}$. As the media type is already provided in the resource proxy list of the CMDI file, there is no component including it. For each LO in the CMDI's resource proxy list, the profile allows the provision of various and multiple checksums, each specifying the algorithm in an attribute.

\subsection{Provenance}

The CMDI 1.2 specification (CMDI Taskforce 2016) implies that provenance information is not to be included in the CMDI file, but instead in one ore more separate file(s) called journal files. One reasoning behind this is that provenance information is not (necessarily) to be machine-interpretable and should be directed to human readers. We implement the journal file in HTML with microformats, as this approach allows formatting for human consumption by means of a web browser and aims at semantic interoperability. We include information in the journal file which is useful both for ensuring reproducibility of research and for keeping track of the development of a resource.

We include a log of every modification to the $\mathrm{CO}$ described by the signpost. These changes contain the following: creation, ingest, injunction, and migration. Moreover, all changes are dated with a time stamp and include a short human-readable log message. We suggest to also include modifications of LOs, i.e. object changes. Changes of the LO are marked as an addition, a replacement, or a removal. An xml:id (Marsh, Veillard and Walsh 2005) attribute can be used in the signpost to identify LOs. This way, the log allows to determine the lifespan of a LO in a machine-readable way.

\footnotetext{
${ }^{6}$ https://curate.acdh.oeaw.ac.at/
} 


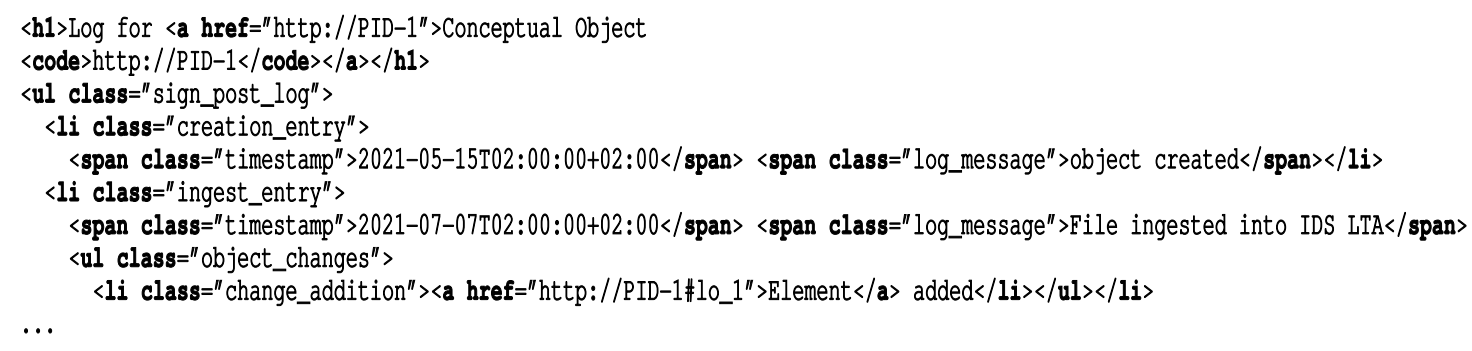

\subsection{PIDs}

The usage of persistent identifiers differs significantly from the current usage in repositories. Traditionally, care is taken to assure that links to logical objects remain available and persistent; COs are not necessarily represented. We reverse this: Not the LO (file) but the CO (signpost) is primary. This means, only the signpost is granted a persistent identifier, and the access to logical objects is through URLs for which the archive does not give any guarantees. As this is currently unconventional, we must alert the user to the impermanence of LO URLs. We have considered the following strategies:

We can implement a notice at the presentation layer of the repository: e.g. using a link text line temporary download link. This would inform human users, but is of no consequence for machine-processing of CMDI records. We suggest this is not a grave problem, for two reasons: First, as long as URLs for logical objects are not reused, tools relying on the ResourceProxyList and even caches will have no problems. This means that for a tool like the CMDI Explorer (Arnold, Campbell et al. 2020) currently developed by CLARIN-D and the CLARIN ERIC, and which will recursively process chains of CMDI records, nothing changes, except there is one link more in the chain for each conceptual object. Moreover, in case of multiple active LOs, the CMDI Explorer would have to avoid downloading them all. Secondly, we assume that by the time signposts are in widespread use, tool authors will have been made aware of the concept, and will take care not to download LOs blindly, but rely on signposts. It may be advantageous to integrate licensing information per LO, potentially in tandem with access control lists. Crawling of resources rather than metadata (including signposts), etc., can be prohibited in the robots.txt.

Alternatively or additionally, one could take care to generate temporary links to logical objects and hence force users to not rely on their URLs. We assume that this strategy generally wastes resources and should only be the last resort.

\section{Signposts for CLARIN: Making Virtual Collections Future-Proof}

Virtual collections are an important asset of the CLARIN infrastructure. They allow to recombine existing collections and individual resources into new collections, which is done in CLARIN's Virtual Collections Registry (https://collections.clarin.eu/, see Elbers 2017). In that sense, they correspond to the case discussed as 'complex corpus structures' above, but with the additional twist that they are potentially defined across repositories. While not a goal in the design of signposts, signposts come in handy when devising virtual collections.

The goal is that such collections are from the user's point of view indistinguishable from non-virtual collections. Tools like the CMDI Explorer allow to work with virtual collections, and generally have to resolve the components of a virtual collection according to the ResourceProxyList and the relationships codified in the Component Metadata of the collection.

Tools using virtual collections have even less control over what happens to constituents. In that sense, the benefit of using signposts is even greater. Consider the traditional use of PIDs in virtual collections as depicted in figure 3: The PIDs used in the virtual collection point to resources directly, also to parts of existing collections. When an item becomes unavailable (red item), the PID will just cause an error. It will not even be easy to decide whether this is just a temporary network issue or a change in the repository.

Now, we introduce signposts. Figure 4 shows what a collection looks like in the traditional way and then what it looks like using signposts. In figure 5, it is shown how using signposts simplifies working with virtual collections: As the single resources are protected behind signposts, the error can be caught immediately, and deliberate changes to the resource will be traceable in time due to the log associated with the signpost. Similar, format migrations can be taken advantage of using the multiple logical objects pointed to by the signpost in such a case. 
Collection A

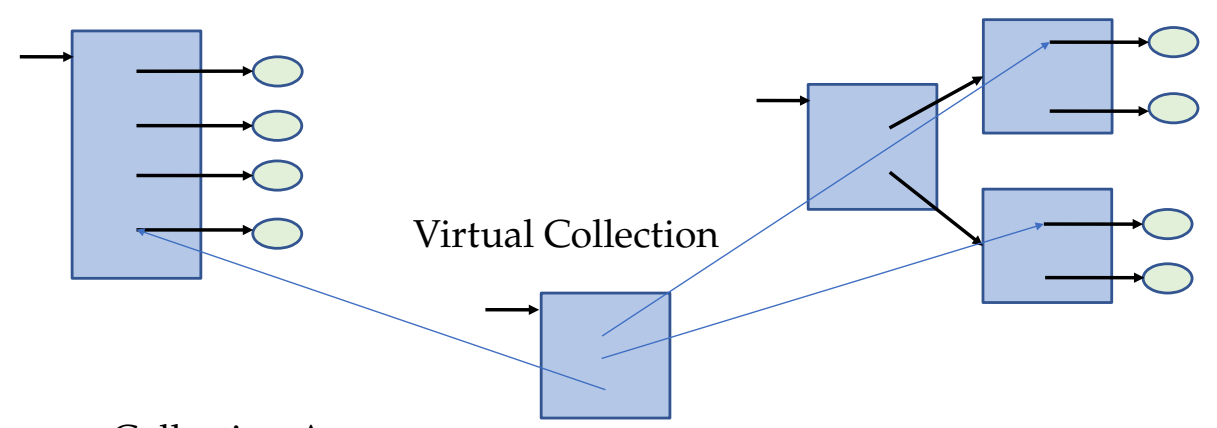

Collection A

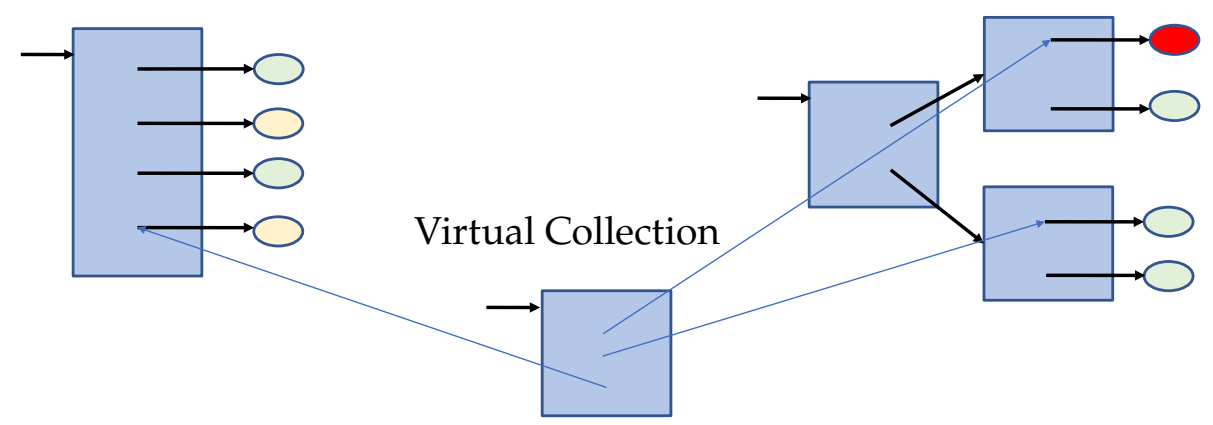

Figure 3: Virtual collection; big boxes are (virtual or non-virtual) collections, ovals are single resources, black arrows are links through PID. The lower collection contains a item (red) which is unavailable.

Collection A

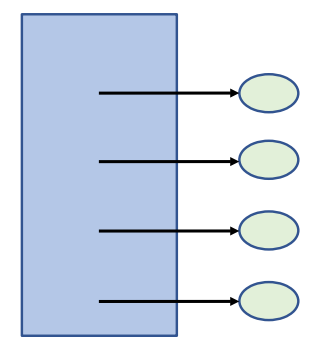

Collection A

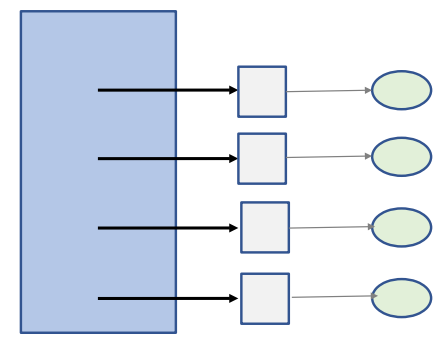

Figure 4: Collection without signposts (left) and using signposts (right); big boxes are (virtual or non-virtual) collections, ovals are single resources, black arrows are links through PID. Small boxes are signposts, light arrows are feeble non-permanent links.

\section{Collection A}

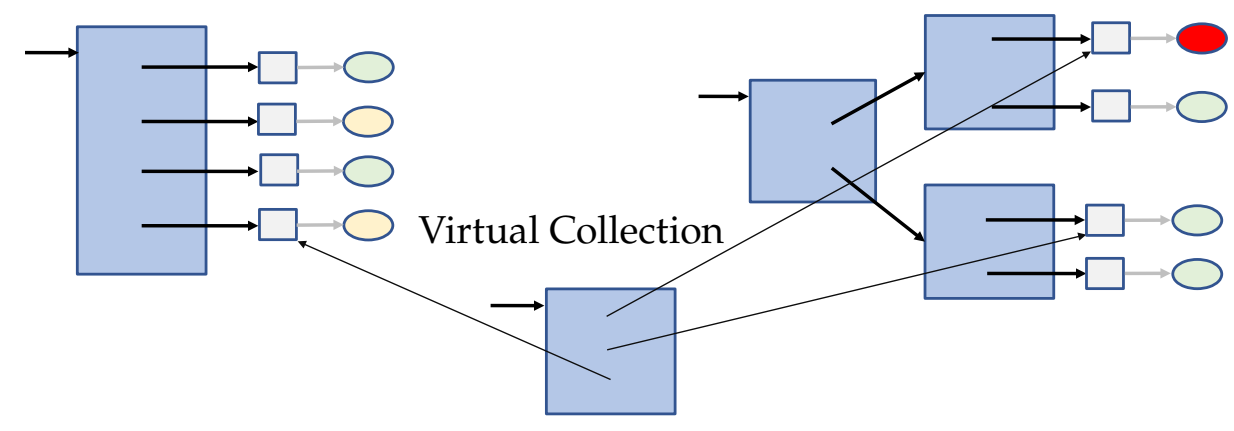

Figure 5: Virtual collection using signposts; big boxes are (virtual or non-virtual) collections, ovals are single resources, black arrows are links through PID. Small boxes are signposts, light arrows are feeble non-permanent links. The collection contains a item (red) which is unavailable. 
Earlier, when referenced data was required to undergo any form of editing, the resources of the virtual collection became unavailable without notice to a user of the virtual collection, besides maybe a webserver error message, but even that would not have been available when a tombstone was in place. Signposts on the other side provide information to a user of a virtual collection that is meaningful, pointing to the actual data or informing them on new versions or added restrictions. This method is scalable in contrast to technical solutions automatically verifying the persistent availability of resources that are part of virtual collections. It also extends to those resources with restricted access, which would not allow an automatic evaluation, for example by comparing checksums of data streams part of a virtual collection.

\section{Signposts in CLARIN: A Corpus and A Virtual Collection}

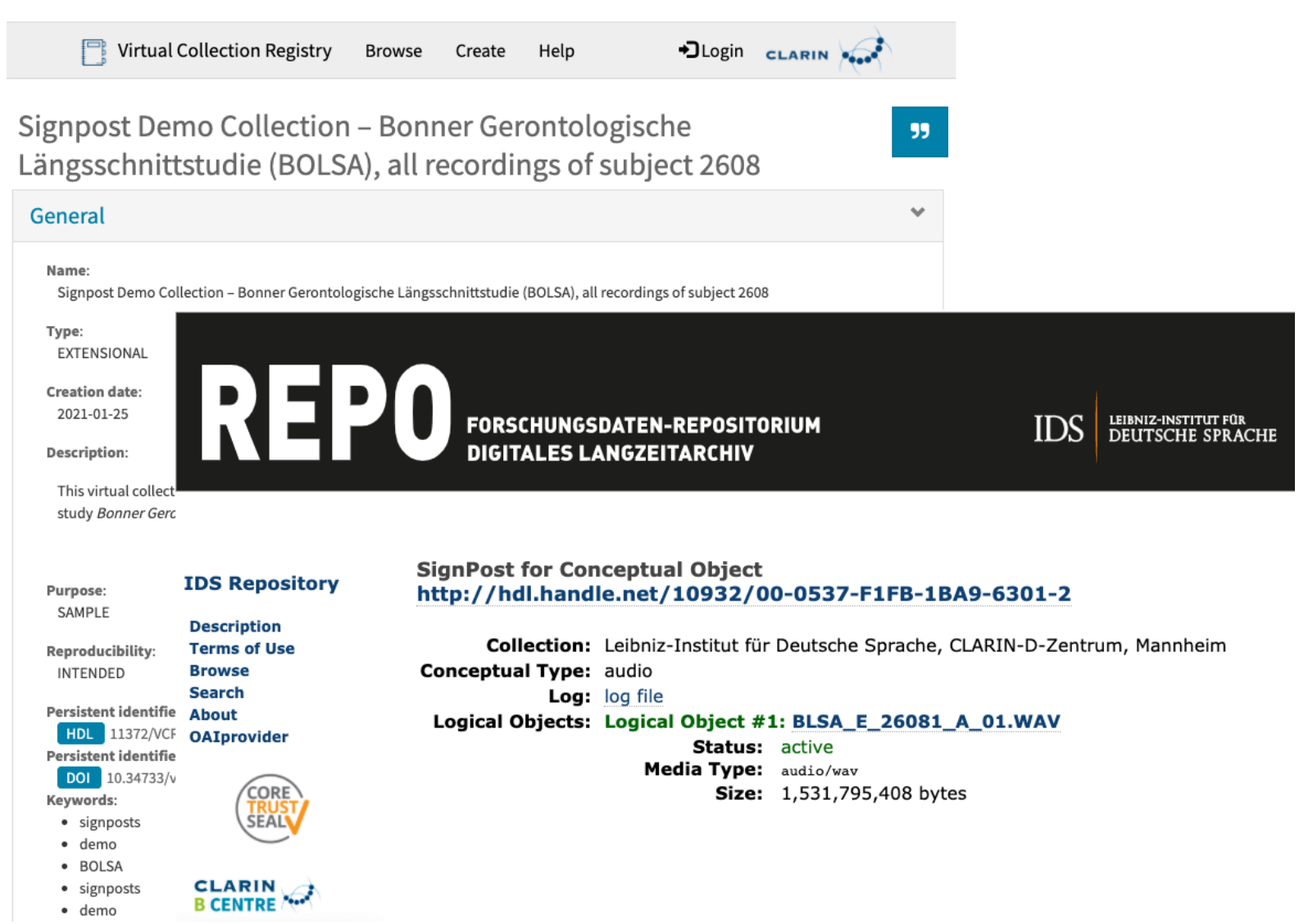

Figure 6: Screenshots from the Virtual Collection Registry showing the virtual collection of all recordings of of subject 2608 .

The first collection that was ingested into a CLARIN repository using signposts was the Bonn Gerontological Longitudinal Study (Bonner Gerontologische Längsschnittstudie, acronym BOLSA, see Lehr and Thomae 1987), which can be viewed in the IDS repository at http://hdl.handle.net/10932/ 00-0537-F2C9-1120-E101-9. This is the audio part of BOLSA, the further data and background information are available at the University of Halle (https://bolsa.uni-halle.de/) It contains interviews with the same 222 subjects (born between 1890 and 1895 and between 1900 and 1905, respectively) between 1965 and 1984.

The data is structured as follows: The whole audio corpus is structured into recording events which are grouped into 'waves' and also associated with test subjects. Recording events contain metadata about the recording (date, place, topics and keywords) as well as the test subject and interviewer. This specific metadata is kept as payload, but all metadata corresponding to facets used by the Virtual Language Observatory are made available as Component Metadata. However, only the whole data set is displayed in the VLO, not the recording events.

The collection and the recording events have been assigned Component Metadata as usual, pointing to their parts through PIDs. All single recordings and all original metadata files have been assigned PIDs which point 
to signposts. These signposts point to the data. Currently, unfortunately, the data cannot be accessed publicly yet for legal reasons.

We extensionally defined a virtual collection (see DOI 10.34733/vcr-1036) according to metadata criteria for all recordings of subject 2608. Screenshots from the Virtual Collection Registry can be found in figure 6 .

\section{Conclusion}

We proposed the notion of signpost for addressing data removal, migration and deduplication in long-term archival of resources, with a specific focus on growing corpora. We also presented the added value to virtual collections and provided an implementation of the concept in the CLARIN infrastructure. The concept of signposts fits to the overall architecture of managing resources in distributed environments, including decentralized provision of metadata endpoints for harvesting, adapted metadata schemas according to ISO 24622-1 (CMDI) and persistent identification. In addition to these features it also allows for consistent integration within Virtual collections as for example implemented in the Virtual Collection Registry (VCR) of CLARIN.

We illustrated the usage of signposts within CLARIN building a virtual collection based on the first collection ingested into a CLARIN repository using signposts.

It is important to note that both from the depositor's and from the end user's point of view, signposts need not be taken into account once repositories and tools implement them. Generating signposts is best done during the ingest of data into the repository, as the are tied to the preservation policy of the repository.

We welcome feedback on the concept and on the implementation. Future work will concern the adaptation of the format, and the integrations with tools, as outlined above.

\section{Acknowledgements}

The work reported here was funded by the German Federal Ministry of Education and Research (BMBF), the Ministry of Science, Research and Art of the Federal State of Baden-Württemberg (MWK), Project Management Agency German Aerospace Centre (DLR), and CLARIN-D.

We thank the anonymous reviewers for helpful comments that have allowed us to sharpen the text.

\section{References}

Arnold, Denis, Ben Campbell, Thomas Eckart, Bernhard Fisseni, Thorsten Trippel and Claus Zinn (2020). 'The CMDI Explorer'. In: Proceedings of CLARIN Annual Conference 2020.05 - 07 October 2020, Online Edition. Ed. by Costanza Navarretta and Maria Eskevich, pp. 157-161. uRL: https : / / office.clarin . eu/v/CE-2020-1738-CLARIN2020_ConferenceProceedings.pdf.

Arnold, Denis, Bernhard Fisseni, Paweł Kamocki, Oliver Schonefeld, Marc Kupietz and Thomas Schmidt (2020). 'Addressing Cha(lle)nges in Long-Term Archiving of Large Corpora'. In: Proceedings of the LREC 2020 Workshop 'Challenges in the Management of Large Corpora' (CMLC-8). Marseille, France.

Marsh, Jonathan, Daniel Veillard and Norman Walsh (Sept. 2005). xml : id Version 1.0. W3C Recommendation TR xml-id. The World Wide Web Consortium. uRL: https://www.w3.org/TR/xml-id/.

Broeder, Daan, Menzo Windhouwer, Dieter Van Uytvanck, Twan Goosen and Thorsten Trippel (2012). 'CMDI: a component metadata infrastructure'. In: Describing LRs with metadata: towards flexibility and interoperability in the documentation of LR workshop programme. Vol. 1.

Burnard, Lou and Syd Bauman, eds. (2020). Guidelines for Electronic Text Encoding and Interchange. TEI P5. version 1.0.0 2007; latest release 4.0.0 on 2020-02-13. Chicago, New York: Text Encoding Initiative.

CCSDS (2012). Reference model for an open archival information system (OAIS). CCSDS 650.0-M-2. 2nd ed. Washington: CCSDS. uRL: https://public.ccsds.org/pubs/650x0m2.pdf. 
CMDI Taskforce (2016). Component Metadata Infrastructure (CMDI): Component Metadata Specification. version 1.2. Tech. rep. CLARIN ERIC. uRL: https://office.clarin.eu/v/CE-2016-0880-CMDI_12_ specification.pdf.

De Smedt, Koenraad, Dimitris Koureas and Peter Wittenburg (2020). 'FAIR Digital Objects for Science: From Data Pieces to Actionable Knowledge Units'. In: Publications 8.2.

Elbers, Willem (2017). Virtual Collection Registry v2. Tech. rep. CLARIN ERIC.

ISO8879:1986 (1986). Information processing - Text and Office Systems - Standard Generalized Markup Language (SGML). Standard No. ISO 8879:1986. International Organization for Standardization.

Kupietz, Marc, Cyril Belica, Holger Keibel and Andreas Witt (2010). 'The German Reference Corpus DeReKo: A Primordial Sample for Linguistic Research'. In: Proceedings LREC'10. Ed. by Nicoletta Calzolari, Khalid Choukri, Bente Maegaard, Joseph Mariani, Jan Odjik, Stelios Piperidis, Mike Rosner and Daniel Tapias. Valletta/Paris: European Language Resources Association (ELRA), pp. 1848-1854.

Lehr, Ursula and Hans Thomae, eds. (1987). Formen seelischen Alterns. Ergebnisse der Bonner gerontologischen Längsschnittstudie (BOLSA). German. Stuttgart: Enke.

Lüngen, Harald and Christopher Michael Sperberg-McQueen (2012). 'A TEI P5 Document Grammar for the IDS Text Model'. In: Journal of the Text Encoding Initiative 3, pp. 1-18. uRL: http: // jtei . revues.org/508.

Neuroth, Heike, Achim Oßwald, Regine Scheffel, Stefan Strathmann and Mathias Jehn, eds. (2009). nestor Handbuch. eine kleine Enzyklopädie der digitalen Langzeitarchivierung. Version 2.0 [3/2010]. nestor.

Sperberg-McQueen, Christopher Michael and Lou Burnard, eds. (1999). Guidelines for Electronic Text Encoding and Interchange. TEI P3. initial release 1994-05-16; last version dated May 1999. Chicago, New York: Text Encoding Initiative. 